\title{
La igualdad de género en la cooperación española y en la iniciativa Marca España. Abordajes e inconsistencias en tiempos de crisis ${ }^{1}$
}

\author{
Julia ESPINOSA FAJARDO \\ Universidad Complutense de Madrid \\ jespinos@ucm.es
}

Recibido: 14.07 .2014

Aceptado: 08.01.2015

\begin{abstract}
RESUMEN:
Desde la década de los noventa, el desarrollo humano y la igualdad de género se reconocen como prioridades de la agenda internacional y así han sido asumidas por el Estado español. Sin embargo, en la actual situación de crisis internacional, y ante la agenda de estabilidad macroeconómica y austeridad fiscal definida en los países ricos, la promoción del desarrollo humano parece quedar en un segundo nivel. Asimismo, los avances logrados a escala internacional en materia de promoción de la igualdad de género desde las políticas públicas también corren el riesgo de verse seriamente afectados.

En el caso de la política exterior y de cooperación española, la ayuda oficial al desarrollo experimenta recortes que la sitúa en cifras de comienzos de la década de los ochenta a la par que la iniciativa Marca España gana centralidad. Todo esto en un escenario donde, de modo generalizado, se asiste a una marginación de los contenidos de género en la agenda pública.

Este artículo analiza en qué modo existe una apuesta por un desarrollo humano pro-equidad de género, en línea con los compromisos internacionales asumidos por el Estado español, en la política de cooperación española y en la iniciativa Marca España en el período 2008-2014. Dado el impacto de ambas políticas en el desarrollo de países del Sur, indaga en si la igualdad de género se incluye o excluye como objetivo en ambas políticas, cómo se define el problema de la desigualdad de género y cuál es el enfoque adoptado y los recursos -económicos e institucionales- existentes para promoverla. Todo ello persigue identificar posibles transformaciones acaecidas en el período de análisis, marcado por la crisis internacional y el cambio de gobierno, así como las inconsistencias entre ambas políticas que pueden limitar el impacto del trabajo pro-equidad de género.
\end{abstract}

Palabras clave: Igualdad de género; Desarrollo; Marca España; Política de cooperación internacional.

${ }^{1}$ El presente artículo se nutre de la investigación "Coherencia de Políticas para el Desarrollo y género" llevada a cabo por Julia Espinosa Fajardo y Begoña Gallardo García para la Plataforma 2015 y más con financiación de la Agencia Española de Cooperación Internacional para el Desarrollo en el marco del Convenio de Educación para el Desarrollo 10-117 "Investigación aplicada, comunicación social y participación ciudadana para el desarrollo humano, mediante la promoción de la coherencia de políticas públicas basadas en los Derechos Humanos, en la equidad de género, en el respeto al medio ambiente y en el comercio responsable". Dicha investigación está publicada por la Plataforma con el título Coherencia de políticas y género. Un análisis feminista de la promoción del desarrollo internacional. 


\title{
The gender equality in the Spanish cooperation and the initiative Marca España. Boardings and inconsistencies in times of crisis
}

\begin{abstract}
Since the nineties, human development and gender equality have become two central priorities of the international agenda and for the Spanish State. However, in the current international economic and financial crisis, and linked to agenda of macroeconomic stability and fiscal austerity set in rich countries, the promotion of human development seems to be on a second level. In this scenario, the progress made internationally in the promotion of gender equality through public policies is also at risk of being seriously affected.

In the case of the foreign and cooperation policy of Spanish State, the official development aid is cut back to figures as in early eighties and, at the same time, the 'Marca España' initiative takes centrality. And, all of this in a scenario where we are attending a marginalization of gender content in the public agenda.

This article looks at if there is a commitment to a pro-gender equality development according to the international commitments of the Spanish State. It specifically analyses if this commitment exists in the 'Marca España' initiative and in the Spanish cooperation policy for the period 2008-2014. Due to the impact of both policies in the development of Southern countries, it investigates whether gender equality is included or excluded as an objective in these policies, the different approaches adopted in this regards and the economic and institutional resources to promote gender equality. All this aims to identify possible transformations occurring in the analysis period, marked by the international crisis and the change of government, and the inconsistencies between the two policies that can limit the impact of the pro-gender equity efforts.
\end{abstract}

Key words: Gender equality; Development, Marca España; International cooperation policy. 


\section{INTRODUCCIÓN}

Diferentes han sido los pasos dados en el reconocimiento de la igualdad de género como un requisito previo para el desarrollo en las últimas décadas tanto en la agenda internacional como por parte del Estado español. Aunque aún queda mucho camino por recorrer, la Convención sobre la eliminación de todas las formas de discriminación contra la mujer (CEDAW) de 1979 y IV Conferencia Mundial sobre Mujeres de Naciones Unidas celebrada en Beijing en 1995 constituyen importantes hitos en la apuesta por eliminar efectivamente la discriminación contra las mujeres, por una parte, y abordar las causas estructurales de la desigualdad de género, por otra. En el marco del Estado español, el compromiso con la igualdad de género queda plasmado en la Constitución española (1978) y en la Ley Orgánica para la igualdad efectiva de mujeres y hombres 3/2007. En materia de desarrollo internacional, además, este compromiso se pone de manifiesto en la ratificación por parte del Estado español de la CEDAW (1984), su protocolo facultativo (2001) y de la Plataforma para la Acción de la Conferencia de Beijing (1995) así como en la Ley de Cooperación Internacional 23/98.

Ahora bien, en el actual contexto de crisis internacional y de políticas de estabilidad macroeconómica y austeridad fiscal impulsadas por los países ricos, la apuesta por un desarrollo humano pro-equidad de género parece no abordarse en la agenda internacional con toda la profundidad que requeriría y, a este respecto, los avances alcanzados hasta el momento pueden verse afectados en gran medida. Justo en pleno proceso de debate sobre la Agenda de Desarrollo Post 2015, la igualdad de género y el empoderamiento de las mujeres son reconocidos como una prioridad (Grupo de trabajo de Naciones Unidas para la Agenda de Desarrollo Post 2015, 2012). Sin embargo, las voces de las organizaciones feministas ponen sobre la mesa la necesidad de un enfoque transformador de género que se centre en las causas estructurales y sistémicas de la desigualdad, que dirija la mirada a los derechos humanos y no sólo al mero crecimiento económico (Women's Coalition, 2014; AWID, 2013; Grupo Mayor de Mujeres, 2013; Moro-Coco y Raaber, 2013).

En el escenario estatal, frente al compromiso con la igualdad de género de los gobiernos de Rodríguez Zapatero (2004-2011), en la actualidad asistimos a una marginación de los contenidos de género en toda la agenda pública y a unas medidas del Gobierno popular que están teniendo un impacto sobre los derechos de las mujeres y están contribuyendo a reproducir la desigualdad estructural de género. Reflejo de ello son el desmantelamiento de la Ley de Atención a la Dependencia, los recortes en sanidad y educación pública así como la reducción de fondos destinados a la lucha contra la violencia de género (Espinosa y Gallardo, 2013 y Fernández-Layos, 2013). 
En este contexto, ¿cómo ha sido y es abordada la igualdad de género en el marco de la política exterior y de cooperación española desde el inicio de la crisis? ¿Existe un alineamiento con los compromisos internacionales en materia de género asumidos por el Estado español? El presente artículo analiza si la igualdad de género ha sido y es una prioridad, cómo se ha definido el problema de la desigualdad de género, cuál ha sido y es el enfoque adoptado y cuáles los recursos económicos e institucionales- con que se ha contado para promoverla en el período 2008-2014. Con ello persigue ver los impactos de la crisis, por una parte, y las transformaciones acaecidas en estas políticas con el cambio de gobierno en 2011, por otra. En concreto, el artículo estudia la política de cooperación y la iniciativa Marca España asumiendo la necesidad de analizar la coherencia de políticas para el desarrollo - promovida por Naciones Unidas, la Organización para la Cooperación y el Desarrollo Económico y la Unión Europea, entre otros agentes ${ }^{2}-$ y dada la centralidad de la iniciativa Marca España en la actual política exterior del gobierno. En este sentido, persigue analizar las consistencias e inconsistencias existentes entre ellas en relación a los compromisos internacionales de género asumidos por el Estado español y reiterados por el presidente Mariano Rajoy en su apuesta por la igualdad entre hombres y mujeres en la $68^{\circ}$ Asamblea General de Naciones Unidas ${ }^{3}$.

Para todo ello, el artículo se apoya en el análisis crítico de marcos ${ }^{4}$ como metodología para revelar los distintos modos de concebir la igualdad de género y

${ }^{2}$ La experiencia internacional ha puesto de manifiesto que los avances en términos de desarrollo no pasan sólo ni fundamentalmente por las políticas de cooperación sino que se requieren políticas integrales y coordinadas. A este respecto, la coherencia de políticas para el desarrollo ha emergido con fuerza en la agenda internacional ante la necesidad de una mayor interrelación y consistencia entre la política de cooperación y el resto de políticas con impactos en terceros países y un mayor alineamiento de estas políticas con los compromisos internacionales en materia de desarrollo (Espinosa y Gallardo, 2013 y Millán et al., 2012).

3 En concreto, el presidente Mariano Rajoy afirmó: "Continuaremos impulsando los objetivos de ONU Mujeres tras un primer año cargado de trabajo y resultados. En este sentido, España, que ha sido el mayor donante de la entidad en su primer año de andadura, mantiene su compromiso con la igualdad entre hombres y mujeres, y con los esfuerzos para poner fin a la violencia contra las mujeres y las niñas". Puede consultarse el discurso completo en https:/www.unric.org/es/actualidades-/358-discurso-de-mariano-rajoy-en-laasamblea-general-de-la-onu

${ }^{4}$ El "análisis crítico de marcos" se concibe "como el estudio de los "marcos interpretativos" de política tal y como se utilizan en la (re)construcción y negociación de la realidad por parte de los actores sociales y políticos en sus respectivos discursos" (Bustelo y Lombardo, 2007: 20). En este sentido, el "análisis crítico de marcos" se centra en el estudio de los diversos discursos políticos existentes dentro de la comunidad política y contribuye a poner al descubierto la diversidad y la competencia entre las interpretaciones de los problemas públicos así como la inconsistencia de las políticas públicas. 
los enfoques existentes para promoverla en las políticas examinadas así como para mostrar las principales ausencias e inconsistencias en materia de igualdad de género. Concretamente, se basa en un análisis de la normativa nacional ${ }^{5}$ en materia de cooperación y Marca España atendiendo a un conjunto específico de preguntas guía $^{6}$ relativas a quién tiene y no tiene la voz; a cómo se define el problema de falta de desarrollo y cómo el problema de la desigualdad de género; y qué tipo de estrategias y acciones se promueven. De forma paralela, el artículo se basa en el análisis de los fondos destinados a igualdad de género así como de la ubicación de las cuestiones de género en los diferentes organigramas a partir de consultas a bases de datos y entrevistas a responsables, tanto de la iniciativa Marca España como de la cooperación española ${ }^{7}$.

A continuación, como punto de partida, se presenta cómo la igualdad de género ha sido incorporada como un compromiso internacional y del Estado español. A partir de ahí, el análisis se centra de forma específica en la política de cooperación española, por un lado, y en la iniciativa Marca España, por otra. En concreto, se examina el abordaje de la igualdad de género y el grado de alineamiento de estas políticas con los compromisos internacionales en esta materia. El artículo se cierra con un capítulo de conclusiones donde se ponen de manifiesto las (in)consistencias entre ambas políticas en relación a los compromisos internacionales de género y cómo éstas se ligan a la crisis y/o al cambio de gobierno.

\section{LA IGUALDAD DE GÉNERO, COMPROMISO INTERNACIONAL Y DEL ESTADO ESPAÑOL}

La igualdad de género constituye, hoy por hoy, un reafirmado compromiso internacional y del Estado español. En efecto, la igualdad de género ha sido reconocida como un objetivo declarado de la comunidad internacional y de este modo ha sido asumido por el Estado español con su firma y adhesión a diferentes compromisos internacionales en esta materia que a continuación se recogen. No obstante, el abordaje de la igualdad de género como prioridad política ha diferido a lo largo de las últimas décadas, primando diferentes tipos de enfoques en línea con

\footnotetext{
${ }^{5}$ Pueden consultarse los documentos relativos a la normativa nacional analizada al final del artículo.

${ }^{6}$ Las preguntas guía que han estructurado el análisis se encuentran recogidas como anexo.

${ }^{7}$ De forma específica, las siguientes personas fueron consultadas: José Ángel López Jorrín, director de Oficina de Alto Comisionado del Gobierno para la Marca España; Elisa Nieto Maestro, Jefa de la Unidad de Género de la Agencia Española de Cooperación Internacional para el Desarrollo; y Nava San Miguel, responsable del Área de Género y Derechos Humanos del Ministerio de Asuntos Exteriores y Cooperación.
} 
distintos modos de comprender el problema de la desigualdad de género y de abordarlo. Grosso modo, desde un enfoque de Mujeres en Desarrollo (MED), el problema de la desigualdad se ha asociado con la discriminación contra las mujeres y su exclusión de los procesos de desarrollo. A la par, desde un enfoque de Género en el Desarrollo (GED), este problema se ha vinculado con las desiguales relaciones de poder existentes entre los géneros que se traducen en diferentes oportunidades, experiencias y beneficios. Como puede verse en la tabla, estas distintas comprensiones de la desigualdad se han traducido, asimismo, en diversas acciones para combatirla: acciones centradas en la integración de las mujeres en los procesos de desarrollo, desde un enfoque MED; y acciones orientadas a cambiar las bases estructurales de la desigualdad de género -con un foco en los mecanismos institucionales y en la capacidades de las mujeres-, desde un enfoque GED (Espinosa, 2011).

Tabla. Enfoques ligados a la promoción de la igualdad de género

\begin{tabular}{|c|c|c|}
\hline Enfoque & Concepción de la desigualdad & Acciones impulsadas \\
\hline Enfoque MED & $\begin{array}{l}\text { La desigualdad entre mujeres y } \\
\text { hombres como discriminación } \\
\text { contra las mujeres que impide un } \\
\text { acceso igualitario a los procesos } \\
\text { políticos y a los beneficios del } \\
\text { progreso social. }\end{array}$ & $\begin{array}{l}\text { Acciones orientadas a promover la } \\
\text { participación activa de las } \\
\text { mujeres en la esfera pública a } \\
\text { través de medidas específicas } \\
\text { para mujeres. }\end{array}$ \\
\hline Enfoque GED & $\begin{array}{l}\text { La desigualdad entre mujeres y } \\
\text { hombres se vincula con causas } \\
\text { múltiples y entretejidas que crean } \\
\text { una relación desigual entre los } \\
\text { sexos. }\end{array}$ & $\begin{array}{l}\text { Acciones que impulsan } \\
\text { procedimientos y mecanismos } \\
\text { institucionales para hacer visibles } \\
\text { las estructuras y procesos } \\
\text { sociales que potencian las } \\
\text { desigualdades de género. } \\
\text { Acciones centradas en potenciar las } \\
\text { capacidades de las mujeres y su } \\
\text { implicación activa en procesos } \\
\text { de toma de decisión. }\end{array}$ \\
\hline
\end{tabular}

Fuente: Elaboración propia.

Dirigiendo la mirada a los compromisos internacionales en sí, la Carta Internacional de Derechos Humanos (1948) constituye el punto de partida en el reconocimiento de que todos los seres humanos nacen libres e iguales y de que es 
necesario impulsar la no discriminación por razón de sexo. Empero, hubo que esperar hasta 1979 para que se aprobase la Convención sobre la eliminación de todas las formas de discriminación contra la mujer (CEDAW) que supuso el compromiso - por parte de todos los Estados firmantes- con la reformulación de leyes para eliminar efectivamente la discriminación contra las mujeres. Y, hasta los noventa, para que en la Conferencia de Viena (1993) los derechos de las mujeres se reconocieran como derechos humanos y en la Conferencia de El Cairo (1994) se asumiera la necesidad de reconocer y proteger los derechos sexuales y reproductivos de mujeres y hombres.

No obstante, el principal hito en relación a los compromisos internacionales de género fue la IV Conferencia Mundial sobre la Mujer de Naciones Unidas (1995), conocida como Conferencia de Beijing. Esta conferencia supuso el reconocimiento del enfoque GED frente al enfoque MED que había predominado con anterioridad. En este sentido, se pasó de poner el foco exclusivo en la discriminación contra las mujeres a también mirar a las relaciones de género y las causas estructurales de la desigualdad. En esta línea, la Declaración y la Plataforma de Acción de Beijing reconocieron que la igualdad de género es un derecho humano y constituye un requisito previo para el desarrollo y la paz y, en este marco, asumieron que la igualdad entre mujeres y hombres ha de estar en el centro de todas las políticas públicas. Se puso sobre la mesa la necesidad de abordar las raíces de la desigualdad que se basan en desiguales relaciones de poder entre mujeres y hombres -manifiestas en el ámbito económico, social, político y cultural, entre otros- así como de promover el empoderamiento de las mujeres. Y, en esta dirección, se definieron dos estrategias específicas de acción: la "mainstreaming de género"8 y el "empoderamiento de las mujeres".

En cuanto a los Objetivos de Desarrollo del Milenio (ODM), estos supusieron un retroceso respecto a Beijing puesto que el foco se puso en la integración de las mujeres -en su incorporación al mercado laboral, al sistema

${ }^{8}$ La estrategia de mainstreaming de género considera que no es posible avanzar hacia la igualdad si no se producen cambios estructurales en las propias instituciones sociales que reproducen un orden de género injusto. En concreto, supone el compromiso de incorporar la igualdad de género como tema central en la agenda así como de realizar diagnósticos, planificaciones, seguimientos y evaluaciones sensibles al género.

${ }^{9}$ La estrategia del empoderamiento de las mujeres sostiene que las desigualdades entre los géneros encuentran sus orígenes en la subordinación de las mujeres en la familia, la comunidad, el mercado y el Estado. De este modo, esta estrategia se orienta a que las mujeres tomen control de sus vidas y decidan de forma autónoma sobre sus objetivos y estrategias, si bien su finalidad es lograr una mayor equidad en las relaciones entre mujeres y hombres. 
educativo y su participación en parlamentos- y respecto a la Conferencia de El Cairo en su apuesta por la salud materna versus salud sexual y reproductiva ${ }^{10}$. A grandes rasgos, supuso una vuelta a un enfoque MED frente al enfoque GED y a postulados en materia de derechos sexuales y reproductivos anteriores a los noventa. Sin embargo, la Cumbre de los ODM 2010 subrayó que la "igualdad entre los géneros, el empoderamiento de la mujer, su pleno disfrute de todos los Derechos Humanos y la erradicación de la pobreza son esenciales para el desarrollo económico y social" y, en esta dirección, afirmó "la necesidad de la aplicación plena y efectiva de la Declaración y la Plataforma de Acción de Beijing" (ONU, 2010: 3). Asimismo, se comprometió a tomar medidas para cumplir con el marco del Programa de Acción de la Conferencia Internacional sobre la Población y el Desarrollo (El Cairo, 1994) y la CEDAW (1979). En este sentido, con la finalidad de acelerar la consecución del ODM 3 "Promover la igualdad de género y el empoderamiento de las mujeres", se subrayó la necesidad de recuperar la agenda alcanzada en la década de los noventa y que ha sido una referencia de los logros alcanzados en cuanto a compromisos internacionales por parte del movimiento internacional de mujeres. Del mismo modo, se dirigió no sólo a integración de las mujeres sino al cambio en las desiguales relaciones existentes entre mujeres y hombres.

En esta línea, en el actual debate sobre la Agenda de Desarrollo Post 2015, la igualdad de género aparece reconocida como una prioridad. Queda por ver, no obstante, si se abordará atendiendo al carácter sistémico y estructural de la desigualdad, como reclaman las organizaciones feministas, o si, por el contrario, su inclusión se hará siguiendo la estrategia de la eficiencia ${ }^{11}$, propia de un enfoque MED (Women's Coalition, 2014; AWID, 2013; Grupo Mayor de Mujeres, 2013; Moro-Coco y Raaber, 2013). Aunque la igualdad de género ya está en la agenda, el debate aún está entre un enfoque MED o un enfoque GED, entre la mera

\footnotetext{
${ }^{10}$ El concepto "salud materna" se centra en mayor medida en la parte reproductiva y orienta su acción exclusivamente a las mujeres como madres. En este sentido, se encuentra en línea más con un enfoque MED que se centra sólo en el rol reproductivo de las mujeres como bien económico. Por el contrario, el concepto de "salud sexual y reproductiva", asumido internacionalmente desde la Conferencia de El Cairo (1994), se enmarca dentro del enfoque GED y recoge el componente social de la salud, poniendo énfasis en el ejercicio de derechos sexuales y reproductivos, e impulsa el trabajo con ambos sexos (Espinosa Fajardo, 2005).

${ }^{11}$ La estrategia de la eficiencia, inserta dentro del enfoque MED, equipara las desigualdades de género con la desigualdad económica. Asume, en este sentido, que la incorporación de las mujeres al mercado laboral incrementa la posición social y económica de las mujeres así como que deriva en equidad para las mujeres. Además, considera que un desarrollo más eficiente y más efectivo pasa por la incorporación de las mujeres como sujeto económicamente activo (Moser, 1995).
} 
incorporación de las mujeres o la transformación de las relaciones desiguales de poder.

En el caso del Estado español, éste ha ido ratificando y firmando todos los compromisos internacionales arriba mencionados. Veamos, a continuación, en qué medida ha ido alineándose a los mismos en la política de cooperación española y en la iniciativa Marca España en el período 2008-2014, hasta qué punto ha adoptado un enfoque GED o un enfoque MED y qué estructuras, recursos e instrumentos se han destinado para la promoción de la igualdad entre mujeres y hombres.

\section{LA IGUALDAD DE GÉNERO EN LA COOPERACIÓN ESPAÑOLA: EL RIESGO DE UN USO COSMÉTICO}

A lo largo de la última década, la política española de cooperación internacional para el desarrollo ha experimentado importantes transformaciones en su apuesta por la igualdad de género en línea con los compromisos internacionales asumidos por el Estado español en esta materia. Gran parte de estos cambios vinieron ligados a la entrada del PSOE al gobierno en 2004. Así, durante la primera legislatura de Rodríguez Zapatero (2004-2008) y frente al anterior énfasis en la erradicación de la pobreza entendida como carencia de bienes e ingresos, la política de cooperación puso especial énfasis en el carácter multidimensional del desarrollo y en la necesidad de ampliar derechos, oportunidades y capacidades de la población más desfavorecida. En este marco y en línea con la Conferencia de Beijing, la igualdad de género se reconoció como un requisito previo para avanzar en el desarrollo y la reducción de la pobreza. Por otra parte, el énfasis en la igualdad de género dentro de la cooperación también se vio favorecido por la apuesta por la igualdad de género como bandera del Gobierno socialista especialmente en sus primeros años ${ }^{12}$.

Como reflejo de esta apuesta por la igualdad, en estos primeros años, la AOD destinada a igualdad experimentó un sustancial incremento al hilo del aumento generalizado de la ayuda y se avanzó, como se expone más adelante, en el

\footnotetext{
${ }^{12}$ En efecto, durante la primera legislatura socialista, la igualdad de género ganó peso en toda la agenda de Gobierno y así quedó reflejado en la conformación del primer Gobierno paritario de la democracia española y, de forma más sustancial, en la aprobación de la Ley para la igualdad efectiva entre mujeres y hombres (Ley 3/2007, del 22 de marzo) y la Ley de medidas de protección integral contra la violencia de género (Ley 1/2004, de 29 de diciembre), entre otras acciones.
} 
desarrollo de documentos de política en línea con los compromisos internacionales en esta materia (Espinosa y Gallardo, 2013). De forma paralela, los discursos relativos a igualdad pasaron de poner el foco en la integración de las mujeres en los procesos de desarrollo - desde un enfoque MED- a promover una mayor equidad de género impulsando el empoderamiento y los derechos de las mujeres y el mainstreaming -desde un enfoque GED-. Similar discurso, alineado con la Conferencia de Beijing, se observó también en la segunda legislatura y parece mantenerse -al explicitar propuestas de abordaje de la desigualdad de género- en el último Plan Director (2013-2016).

En líneas generales, la igualdad de género ya se definía como principio del desarrollo y como una de las prioridades sectoriales en la Ley 23/1998 de Cooperación Internacional para el Desarrollo (Cirujano Campano, 2005 y BOE, 1998). Sin embargo, no es hasta el II Plan Director de la Cooperación Española 2005-2008 cuando se adopta el denominado enfoque GED identificando la equidad de género como prioridad horizontal; estableciendo un sector específico de "género y desarrollo"; y atendiendo a la desigualdad de género a lo largo de todas líneas de acción propuestas (MAE, 2005). A diferencia del Plan Director anterior, no se trata de que mujeres y hombres tengan sólo las mismas oportunidades, sino que también cuenten con los mismos derechos, libertades, alternativas y responsabilidades. En esta dirección, en el discurso se pasa de promover la igualdad de oportunidades a impulsar la promoción de la equidad de género comprendida ésta como un requisito para el desarrollo y la paz. De igual modo, el foco no se dirige de forma exclusiva a la discriminación de las mujeres, sino a las desigualdades estructurales de género.

En la misma línea, la "Estrategia de Género en Desarrollo de la Cooperación Española" (MAEC, 2007) asume como objetivo general la promoción del pleno ejercicio de los derechos humanos y la ciudadanía de las mujeres como forma directa de incidir en la reducción de la pobreza. Para ello, considera central potenciar la institucionalización del enfoque de género, trabajar el mainstreaming de género en las otras prioridades horizontales y promover el pleno ejercicio por parte de las mujeres de sus derechos económicos, sociales, sexuales y reproductivos, civiles, políticos y culturales. Se pone el foco, por otra parte, en la igualdad formal, la igualdad real, la participación política de las mujeres y su empoderamiento.

El III Plan Director de la Cooperación Española (2009-2012), por su parte, mantiene la estrategia empleada en el anterior plan y se incide en la necesidad de seguir avanzando en la promoción del mainstreaming de género y en el empoderamiento de las mujeres para contribuir a una igualdad real. En este momento, el enfoque GED, aparece reforzado en su parte operativa con los contenidos de la Eficacia de la Ayuda en consonancia con los principios operativos 
de la Declaración de París, recuperando además el enfoque de Derechos Humanos de las mujeres como la mejor forma para luchar contra la pobreza (MAEC, 2009).

En la misma dirección, el último Plan Director de la Cooperación Española (2013-2016) identifica entre sus ocho orientaciones generales una específica -la número 5- centrada en "promover los derechos de las mujeres y la igualdad de género". En concreto, el Plan reconoce que "la crisis económica tiene un impacto especialmente negativo para las mujeres, en términos de acceso efectivo a recursos y a derechos fundamentales" y "la feminización de la pobreza y la discriminación contra las mujeres sigue siendo universal e incuestionable" (MAEC, 2012: 26). Para avanzar en materia de igualdad, propone impulsar la transversalización del enfoque GED y define tres niveles de actuación: líneas para la igualdad formal, líneas para la igualdad real y acciones específicas para el empoderamiento de las mujeres.

Por una parte, las líneas para la igualdad formal persiguen la adecuación de los marcos normativos para avanzar en el cumplimiento de los derechos económicos, laborales, sociales y culturales de las mujeres, así como en materia de derechos sexuales y reproductivos y políticos. Por otra, las líneas para la igualdad real promueven el fortalecimiento de la gobernabilidad y la integración de procesos regionales. Por último, las acciones específicas para el empoderamiento priorizan el fortalecimiento de las organizaciones feministas y de mujeres de la sociedad civil y establecen líneas concretas para paliar todas las formas de violencia y discriminación contra las mujeres y las niñas. Entre las acciones específicas destacan aquéllas orientadas a paliar "discriminación y vulnerabilidad de las mujeres en la economía y el trabajo, especialmente en lo referente a la economía de los cuidados, la situación de las trabajadoras del hogar, la economía informal y las mujeres en las áreas rurales" (MAEC, 2012: 27).

No obstante, a pesar de este importante reconocimiento de la igualdad de género como contenido clave de la cooperación española, las mujeres también son consideradas - desde una estrategia de la eficiencia, inserta en el enfoque MEDcomo agente clave para generar crecimiento económico con su participación activa en el mercado laboral formal. Así, se propone propiciar que "los sectores más empobrecidos, especialmente las mujeres, se integren más intensamente en las actividades generadoras de renta y riqueza" (MAEC, 2012: 23). En este sentido, hay que vigilar que el discurso pro igualdad de género no se convierta en un arma de doble filo que lleve a potenciar los múltiples roles de las mujeres y niñas en tanto que agentes clave para el crecimiento económico y se genere, de este modo, una sobrecarga laboral en las mismas (Pearson, 1999). Esta práctica conllevaría además sacar de la población objetivo a los hombres y niños que tienen un papel central en 
el avance hacia la igualdad de género ${ }^{13}$. No hay que olvidar que el problema de la desigualdad de género se enraíza en relaciones de poder desiguales entre mujeres y hombres (Cirujano Campano, 2005 y Moser, 1995).

En cualquier caso, a grandes rasgos, existe en la cooperación española una apuesta por la promoción de los derechos de las mujeres y la igualdad de género que se reconoce como uno de los objetivos fundamentales de la cooperación española y que no ha perdido centralidad dentro de las orientaciones políticas con el cambio de un gobierno del PSOE a otro del PP, ni como consecuencia de la crisis. Específicamente, y en línea con los compromisos internacionales de género, existe un foco en la feminización de la pobreza, la discriminación contra las mujeres y la transversalización del enfoque de género. Ahora bien, si dirigimos la mirada a las estructuras y recursos existentes, no encontramos cambios sustantivos lo que cuestiona si el compromiso con la igualdad de género está siendo promovido de forma cosmética.

De modo concreto, la pérdida de ubicación de los contenidos de género en el organigrama de la AECID -ligada al cambio de gobierno- supone una transformación en relación a su centralidad dentro de la estructura de la propia agencia. De hecho, con la reforma del Estatuto de la AECID en 2012, la Dirección de Cooperación Sectorial, de Género y ONGD pasa a denominarse Dirección de Cooperación Multilateral, Horizontal y Financiera. Asimismo, se suprime el Departamento de Cooperación Sectorial y de Género y éste pasa a denominarse exclusivamente Departamento de Cooperación Sectorial (BOE, 2012b). Si bien continúa la Unidad de Género -dependiente del Departamento de Cooperación Sectorial de esta Dirección-, lo hace bajo este renombrado departamento lo que revela un cambio formal en la posición de los contenidos de género en relación a los temas prioritarios en cooperación.

Por otra parte, en cuanto al presupuesto de la cooperación española dirigido a cuestiones de género, como puede verse en el siguiente gráfico, en el período analizado se observa una caída de la ayuda oficial al desarrollo (AOD) destinada tanto a organizaciones e instituciones de igualdad, en relación al total de la AOD, como a programas de salud sexual y reproductiva ${ }^{14}$. Independientemente del recorte

${ }^{13}$ Respecto a la centralidad de los varones y los niños en los procesos de empoderamiento de las mujeres y en el progreso hacia la igualdad de género, puede consultarse el proyecto Pathways for Women's Empowerment del Institute of Development Studies: http://www.pathwaysofempowerment.org/

${ }^{14}$ Se han seleccionado este sector y subsector del Comité de Ayuda al Desarrollo (CAD) dada su vinculación a los contenidos de género y la debilidad del "marcador de género" en 
general de la AOD en este período, la ayuda destinada a organizaciones e instituciones de igualdad ha pasado de conformar un 3,14\% del total de la AOD en 2008, a un $2,08 \%$ en $2011^{15}$. Mayor caída se observa en la AOD dirigida a los programas de salud sexual y reproductiva, que en 2008 recibían el $2,10 \%$ del total de la AOD y en 2011 el $0,90 \%$. Así, en términos relativos, se ha recortado aproximadamente un tercio de los fondos destinados a organizaciones e instituciones de mujeres y más de la mitad de la ayuda dirigida a salud sexual y reproductiva, tema especialmente crítico con el Gobierno popular.

Gráfico. AOD española dirigida a programas de salud sexual y reproductiva y a organizaciones e instituciones de igualdad. Porcentaje sobre total de AOD española, 2008-2011.

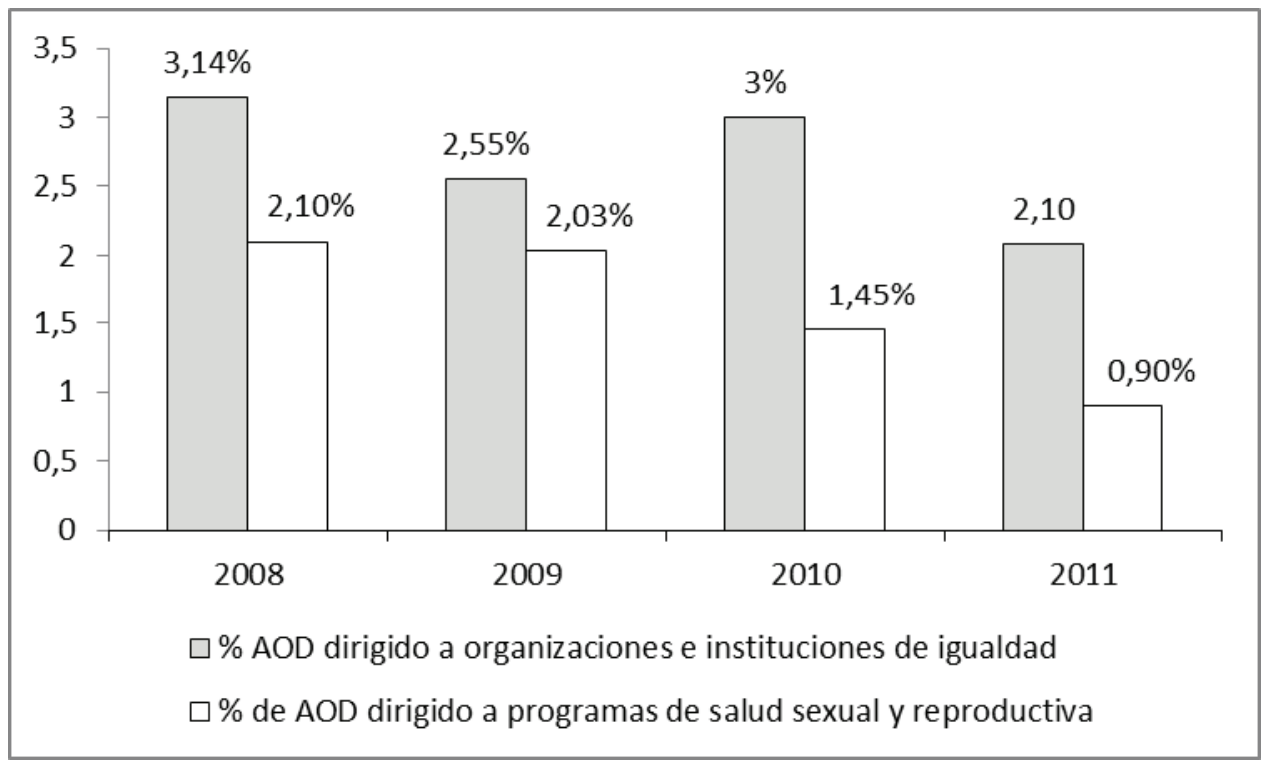

Fuente: Elaboración propia a partir de base de datos del CAD.

tanto que se elabora con la información proporcionada por los Estados sobre la inclusión de género en su AOD.

${ }^{15}$ Los datos sobre AOD española por sectores y subsectores sólo están disponibles hasta 2011 en la base de datos del Comité de Ayuda al Desarrollo. No existe información específica al respecto ni de la ayuda ejecutada en 2012 ni de los compromisos para 2013 y 2014. 
Tal y como ha puesto de manifiesto el estudio llevado a cabo, esta caída comienza ya en la última legislatura socialista y no existen datos públicos al respecto sobre la ayuda ejecutada en 2012 y 2013, ni sobre los compromisos para 2014 para estos sectores. En este punto se observa que la desatención a los compromisos de género ya comenzó en la última legislatura socialista, así como se pone de manifiesto una falta de transparencia por los actuales los responsables gubernamentales de la Cooperación Española.

Por otro lado, aunque no existen cifras oficiales sobre AOD para sectores críticos de género desde 2011, diferentes actuaciones del Gobierno popular reflejan un ataque directo al compromiso oficial con la equidad de género en el campo de la cooperación. Así, por ejemplo, queda de manifiesto en el cierre de la oficina ONU Mujeres en el Estado español a finales de 2012 y la no financiación de la Red de Mujeres Africanas y Españolas -donde participaban unas 1.500 personas y organizaciones de España y más de 50 países africanos-. Todo esto contradice el compromiso con la igualdad entre hombres y mujeres expreso por el presidente Mariano Rajoy en la $68^{\circ}$ Asamblea General de Naciones Unidas en 2013.

Además, los recortes en materia de género pueden poner en peligro la implementación eficaz de las directrices de género para la programación operativa elaboradas desde la Unidad de Género de la AECID, así como del conjunto de herramientas y listas de chequeo definidas por esta misma unidad para seguir avanzando en la transversalización del enfoque de género en los marcos de asociación país, en sectores específicos y en el trabajo con las ONGD. En concreto, la caída de fondos destinados a género supone un limitante para establecer y mantener estructuras y mecanismos de gestión que faciliten una incorporación efectiva de los contenidos de género en la gestión, seguimiento y evaluación de la ayuda española así como una mayor coordinación de actores y actoras en materia de género y desarrollo.

Asimismo, la reducción de AOD dirigida a instituciones y organizaciones de mujeres tiene una implicación significativa para el avance hacia la construcción de una agenda de cooperación que incluya los contenidos críticos de género. Como señala Kabeer, resulta fundamental apoyar la agencia transformadora de las mujeres lo que supone aumentar la capacidad de las mujeres para "preguntar, analizar y actuar sobre las estructuras patriarcales que constriñen sus vidas" (2005: 15). Asimismo, hay que destacar que la incorporación de contenidos de género sin incluir las voces de las mujeres conduce a una descontextualización de las políticas y el fin de las iniciativas destinadas específicamente a la igualdad de género, lo cual es perjudicial para los intereses de las mujeres en los países donde la segregación de género extrema requiere que estas cuestiones sean abordadas de forma específica (Mukhopadhyay, 2007). 
En el actual escenario, los recortes y estrictos programas de austeridad están poniendo en riesgo las acciones de cooperación y las perspectivas de avanzar en materia de desarrollo humano. En efecto, la AOD española en 2013 se situó en el 0,16\% de la renta nacional bruta, frente al $0,7 \%$ reclamado internacionalmente y en cifras que nos sitúan a comienzos de la década de los ochenta. Esta caída es aún más preocupante porque, como señalan Martínez y Martínez, "la AOD que verdaderamente el gobierno decide destinar voluntariamente a promover el desarrollo, adquiere una dimensión insignificante en el conjunto del gasto -un 0,09\%-" (2014: 74). El otro 0,07\% de la AOD corresponde a las contribuciones obligatorias al presupuesto de desarrollo de la Unión Europea y a organizaciones financieras internacionales. Sin embargo, "no se trata únicamente de recortes y ajustes, que es lo que podemos ver en la superficie, sino de un cambio de modelo social en Europa que amenaza con dar al traste con derechos y logros sociales alcanzados y consolidados durante décadas"(Atienza y Hernández, 2012). En este sentido, el compromiso con la igualdad de género -mantenido en el discurso de cooperación del Gobierno popular como hemos expuesto- corre un alto riesgo de evaporarse en la práctica política y más cuando otras políticas -en el área de igualdad, salud sexual y reproductiva, dependencia, entre otras- están vulnerando todo principio de equidad y justicia social suscrito tanto en la legislación nacional como en los acuerdos internacionales (Alonso y Poleo, 2013, Fernández-Layos, 2013, Lombardo, 2013).

\section{LA INICIATIVA MARCA ESPAÑA, UNA INICIATIVA CIEGA AL GÉNERO}

"La Marca España es lo que somos todos los españoles, empresarios, trabajadores, instituciones, administraciones públicas y políticos. Es lo que hacemos día a día, el esfuerzo a través del cual buscamos prosperar como individuos y, por agregación, como sociedad"

(Escuela Diplomática, 2012: 235).

La iniciativa Marca España nace en el año 2002 con la finalidad de sensibilizar a las empresas e instituciones sobre la relevancia de promover la buena imagen del país en el exterior así como con la finalidad de difundir información sobre cómo promocionar esta imagen. Con la entrada al gobierno del PSOE en 2004 , sin embargo, este proyecto pasa a un segundo plano y no vuelve a tener un papel central en la agenda política hasta que el Partido Popular vuelve a tomar el poder a finales de 2011 (Millán, 2013b). La apuesta por la internacionalización de la empresa -impulsada por el último gobierno de Rodríguez Zapatero como una de las vías para la salida de la crisis ${ }^{16}$ - es abordada entonces desde un abanico más

\footnotetext{
${ }^{16}$ Puede consultar más información sobre la apuesta socialista por la internacionalización de la empresa en Espinosa y Gallardo (2013).
} 
amplio: la Marca España que intenta crear y divulgar una imagen-país para fomentar la recuperación económica. En este sentido, el impulso de la Marca España constituye uno de los grandes cambios acaecidos con el paso del último gobierno de Rodríguez Zapatero al actual gobierno de Rajoy.

La apuesta del Gobierno popular por el proyecto Marca España encuentra su máxima expresión en la constitución del Alto Comisionado del Gobierno para la Marca España que, con rango de Secretaría de Estado, es responsable de la planificación, impulso y gestión coordinada de las acciones para promover la imagen exterior de España en el marco de las directrices y estrategias fijadas por el Consejo de Política Exterior (BOE, 2012a). La creación de este organismo, asimismo, ha venido acompañada de una amplia campaña de promoción de la Marca España que incluye la apertura del Portal de Internet Marca España ${ }^{17}$, así como la publicación del libro "Retos de nuestra acción exterior: Diplomacia Pública y Marca España" (Escuela Diplomática, 2012). Al mismo tiempo, la Marca España se ha conformado como uno de los temas centrales en los discursos del actual equipo del MAEC.

En la actualidad, la Marca España constituye una política de Estado cuya garantía de continuidad se liga al consenso por encima de los cambios políticos. Su emergencia se vincula a la necesidad de coordinar la diversidad de sujetos públicos y privados- que desarrollan acciones en el exterior y la variedad de las mismas. A grandes rasgos, su finalidad es mejorar "la imagen exterior de España", así como "los resultados y el logro de contribuciones medibles para los intereses de España en los ámbitos económico, cultural, social, científico y tecnológico" (BOE, 2012a: 46129). En esta línea, se persigue impulsar la diplomacia pública, la diplomacia económica, la cultura española como elemento diferenciador y la denominada diplomacia de los ciudadanos ${ }^{18}$ (Escuela Diplomática, 2012). Para ello, cuenta con una Oficina del Alto Comisionado del Gobierno para la Marca España, sin dotación presupuestaria y donde trabajan dos diplomáticos y cuatro funcionarios administrativos y auxiliares, de acuerdo con la información proporcionada por la propia Oficina. En cuanto a su ubicación en el organigrama, esta Oficina depende

\footnotetext{
17 El enlace a esta página web es: http://marcaespana.es/es/quienes-somos/que-es-marcaespana.php

${ }^{18}$ La diplomacia de los ciudadanos hace referencia al compromiso de los ciudadanos para la configuración de la Marca España, para "lograr que los activos diferenciales que han permitido a muchos de nuestros ciudadanos prosperar sean la norma y no la excepción dentro de nuestra economía" (Escuela Diplomática, 2012: 235). Es una llamada a todos los españoles para hacer "de este país un ejemplo de superación y un caso de éxito en los próximos años” (Escuela Diplomática, 2012: 235).
} 
funcionalmente de la Presidencia del Gobierno y jerárquicamente del Ministerio de Asuntos Exteriores y Cooperación.

A grandes líneas, la iniciativa Marca España asume que la promoción de una buena imagen-país potenciará el "aumento de las exportaciones, la atracción de inversión extranjera a España, el apoyo a la internacionalización de nuestras empresas - potenciando el efecto tractor de las grandes empresas sobre las PYMES que buscan nichos en el mercado exterior-, el incremento del turismo y, en definitiva, la recuperación económica de España" ${ }^{\text {"19. }}$. Además, se considera que el crecimiento económico tendrá de forma directa efectos positivos sobre el "bien común" y, por tanto, la Marca España se define como una herramienta central para abordar la crisis económica y social que caracteriza al Estado español en estas fechas (Escuela Diplomática, 2012).

Esta iniciativa sostiene que una buena imagen-país contribuirá al crecimiento económico y que éste, desde una óptica neoliberal y un enfoque próximo a la teoría de la modernización, traerá consigo el progreso social y el beneficio para todas las personas. Asimismo, iguala el beneficio de la empresa española con el beneficio de la sociedad española en su conjunto. Se apoya, por tanto, en la visión neoliberal de que el mejor modo de promover el desarrollo es promover el laissez faire, el libre mercado. Empero, como los estudios de desarrollo ponen de manifiesto desde la década de los setenta, la promoción del desarrollo precisa de un enfoque que atienda no sólo al crecimiento económico sino también a otras dimensiones que influyen en el mismo como el respeto de los Derechos Humanos, el impulso de la igualdad de género, la protección del medioambiente, el establecimiento de sistemas de participación democrática, entre otras. Requiere, al mismo tiempo, de una atención a la interconexión entre las diferentes partes del planeta y no sólo un foco en el caso español. Por otra parte, la promoción del crecimiento económico en sí no trae consigo un reparto equilibrado de los beneficios. Por el contrario, debido a las relaciones de poder y las desigualdades estructurales existentes -entre ellas las desigualdades de género-, los beneficios recaerán en mayor medida en aquellos grupos cuyos intereses específicos hayan sido atendidos en la definición de las acciones puestas en marcha (Espinosa y Gallardo, 2013).

\footnotetext{
${ }^{19}$ Extraído de la web de la Marca España: http://marcaespana.es/es/quienes-somos/que-esmarca-espana.php
} 
A este respecto, cabe mencionar que junto con las instituciones públicas -el Alto Comisionado del Gobierno para la Marca España ${ }^{20}$, las grandes empresas españolas tienen una importante voz en esta iniciativa. De hecho, el Foro de Marcas Renombradas -alianza entre las marcas líderes y las administraciones y organismos públicos- es definido como interlocutor y socio preferente de la iniciativa Marca España con el objetivo de facilitar la colaboración público-privada. Se considera que "sin Telefónica, Ferrovial, Inditex, Iberdrola, Acciona, Santander, Acerinox, Mango, BBVA y tantas otras empresas, sería realmente difícil sostener una imagen de calidad en el exterior" (Escuela Diplomática, 2012: 98). Sin embargo, la actividad de estas empresas -especialmente en América Latina- no siempre ha potenciado una buena imagen del país (Pérez González y Fanjul, 2012 y Noya, 2009). Y es difícil que esto suceda cuando algunas empresas españolas se han aprovechado de la laxitud o falta de regulación económica, laboral y/o medioambiental para sacar importantes beneficios de países del Sur vulnerando los Derechos Humanos (Intermón Oxfam, 2013). Por otro lado, se asume que a través de las grandes empresas se beneficiará la imagen del conjunto de los productos y servicios españoles $\mathrm{y}$, de este modo, se promoverán los intereses generales del Estado español. Sin embargo, los intereses de las empresas españolas no siempre coinciden -sino que incluso se pueden contraponer-a los intereses de la ciudadanía española. Así se pone de manifiesto, por ejemplo, cuando algunas de las grandes empresas españolas -como Abengoa, Abertis, Acciona, Acerinox, Endesa, Gas

${ }^{20}$ El Alto Comisionado de la Marca España es el principal responsable de la gestión de esta iniciativa y depende funcionalmente del presidente del Gobierno, a través del Consejo de Política Exterior, y orgánicamente del Ministro de Asuntos Exteriores y de Cooperación. Por su parte, el Consejo de la Marca España está conformado -desde noviembre de 2012por la Secretaría de Estado de Comunicación de la Presidencia del Gobierno, el Consejo Superior de Deportes, la Secretaría General de Agricultura y Alimentación (MAGRAMA), la Secretaría General de Política de Defensa (Minisdef), el Comité Olímpico Español, el Instituto Cervantes, el Real Instituto Elcano, el ICEX, TURESPAÑA, la Fundación Carolina, la D.G. de Relaciones Económicas Internacionales (MAEC), la D. G. de Medios y Diplomacia Pública (MAEC), D.G. de Comercio e Inversiones, D.G. de Política Económica (Ministerio de Economía y Competitividad), RTVE, Agencia EFE y Embajador de España para la Diplomacia Cultural.

Junto con el Alto Comisionado y el Consejo de la Marca España, en los grupos de trabajo de asistencia técnica, se incluyen a representantes de los departamentos ministeriales y organismos públicos dependientes de la Administración General del Estado, así como a entidades públicas y privadas que se considere conveniente en atención a los asuntos que se vayan a tratar (BOE, 2012a y Escuela Diplomática, 2012). 
Natural, Repsol, Telefónica, entre otras - evaden impuestos en paraísos fiscales (Observatorio de Responsabilidad Social Corporativa, 2012).

En este marco, ¿hasta qué punto la iniciativa Marca España se encuentra alineada con los compromisos internacionales de género asumidos por el Estado español? En la documentación analizada no existe ninguna referencia a los contenidos vinculados con la igualdad de género (BOE, 2012a y Escuela Diplomática, 2012). Es más, de acuerdo con el director de la Oficina del Alto Comisionado del Gobierno para la Marca España, se considera como un tema donde se ha avanzado lo suficiente y sobre el que no es necesario incidir más. En este sentido, la igualdad de género no constituye un tema en absoluto dentro de la agenda de la Marca España y no existe ningún tipo de estructura, recurso ni mecanismo vinculado a ella. Se desatiende a la estrategia de mainstreaming de género que, emanada de la Conferencia de Beijing, subraya la necesidad de poner la igualdad de género en el centro de la toma de decisiones y a lo largo de todo el ciclo de la política pública reconociendo que -si no se hace así- se tiende a reproducir la desigualdad estructural de género.

Mediante el impulso de la imagen-país se persigue potenciar las exportaciones y el apoyo a la internacionalización de la empresa como dos elementos clave para la recuperación económica del país. Sin embargo, no se presta atención a los posibles impactos -sociales, económicos, medioambientales y de género, entre otros- de las mismas en terceros países. No se reconoce que los efectos de las acciones empresariales son diferentes en mujeres y varones y que son necesarias actuaciones específicas a este respecto. Se pasa por alto que, si no se actúa de forma específica, los beneficios tienden a ser mayores para varones que para mujeres. Como numerosos estudios sobre comercio y género ponen de manifiesto, la liberalización del comercio tiene impactos diferenciados en mujeres y varones por su desigual relación de poder, roles, acceso a recursos, oportunidades y barreras a la participación económica (Salvador, 2012, Women Watch, 2011, Floro y Hoppe, 2005 y Williams, 2003).

En este punto, la iniciativa Marca España es acorde al modelo económico neoliberal reinante en la actualidad que, en muchas ocasiones, es incoherente con los compromisos internacionales en materia de igualdad de género y derechos humanos (Floro y Hoppe, 2005). El foco se centra en maximizar la productividad y la eficiencia y esto supone una priorización de las actividades mercantiles sobre otras relacionadas con la sostenibilidad de la vida que no pasan por el mercado (Jubeto, 2011). A este respecto, no se atiende de forma sistemática a otros criterios clave en materia de economía como son la sostenibilidad, la interrelación entre lo "productivo" y lo "reproductivo" y el uso óptimo de los recursos bajo criterios de justicia. Es más, se sigue considerando que el sujeto central en la economía es un 
ser independiente y autónomo que toma decisiones racionales en función de su interés. No obstante, se ignora la interdependencia existente entre los seres humanos y que ésta se da en condiciones de desigualdad (León, 2008). Se apoya, por consiguiente, un concepto reducido de lo económico donde sólo se incluye lo "productivo" desatendiendo la relevancia de lo "reproductivo" -ámbito tradicional de actividad femenina- para el mantenimiento de la vida y, por ende, para la economía.

Por otro lado, el modelo de economía neoliberal -en que se apoya y que respalda la iniciativa Marca España- se basa en una división sexual del trabajo que sitúa en una posición de exclusión a las mujeres. Las mujeres realizan mayores tareas productivas y reproductivas con menor remuneración, protección y valoración social. Si no se realizan acciones específicas para fomentar un mayor equilibrio en el reparto de tareas y beneficios entre mujeres y hombres, la iniciativa Marca España estará potenciando que se reproduzcan estos esquemas y, lo que es más, se apoyará en estos para sacar ventaja comparativa. Así sucede, como diversas investigaciones han puesto de manifiesto, en sectores con mano de obra muy feminizada -como puede ser el textil- donde las empresas de países del Norte aprovechan una mano de obra muy barata para obtener mayores ganancias, así como mejorar su productividad y su eficiencia (Braunstein y Epstein, 2002 y Berik, Rodgers y Zveglich, 2004 en Floro y Hoppe, 2005).

En línea con la estrategia de promocionar nuestra imagen país en el extranjero, la falta de atención a la igualdad de género en la iniciativa Marca España desaprovecha el reconocimiento internacional que - promovido por los gobiernos de Rodríguez Zapatero- había ganado el Estado español en la promoción de la igualdad de género y los derechos de las mujeres y las niñas y que constituye un gran valor en sí mismo.

Tal y como se pone de manifiesto, el compromiso con la igualdad entre hombres y mujeres -expresado por el presidente Mariano Rajoy en la $68^{\circ}$ Asamblea General de Naciones Unidas en 2013- no está presente en la iniciativa Marca España. Además, y en contra de los compromisos internacionales asumidos en materia de género, la falta de atención a la igualdad de género en esta iniciativa contribuye a reproducir la desigualdad estructural de género.

\section{CONCLUSIONES}

Bajo el paraguas de la crisis internacional, numerosas han sido las políticas promovidas que están poniendo en riesgo el compromiso con un desarrollo humano pro-equidad de género, tanto en el escenario internacional como en el estatal. El predominio de una agenda neoliberal -en muchas ocasiones contraria a la agenda de 
los derechos humanos- está haciendo mella en la apuesta internacional por el desarrollo humano pro-equidad de género en plena definición de la Agenda de Desarrollo Post 2015. A escala estatal, las políticas analizadas revelan diferentes grados de priorización y abordaje de la igualdad de género y ponen de manifiesto una escasa coherencia en relación con los compromisos internacionales de género y desarrollo asumidos por el Estado español y con las declaraciones del presidente Mariano Rajoy en la $68^{\circ}$ Asamblea General de Naciones Unidas. Una incoherencia que, por otra parte, se ha visto favorecida tanto por el contexto de crisis como, en algunas ocasiones, por el cambio de gobierno en 2011.

Ligado a la crisis, la política exterior y de cooperación española ha experimentado dos cambios sustanciales: la caída de la ayuda oficial al desarrollo a cifras que nos sitúan a comienzos de la década de los ochenta del pasado siglo, y la nueva centralidad de la iniciativa Marca España como herramienta del Gobierno Popular para hacer frente a la crisis. Si bien la caída de la AOD se inicia en la última legislatura socialista, con el gobierno de Mariano Rajoy la misma se acentúa. Por otra parte, la apuesta por la Marca España recoge, en cierta medida, la apuesta por la internacionalización de la empresa ya promovida por el gobierno de Rodríguez Zapatero como una de las vías para la salida de la crisis. Sin embargo, constituye una transformación clara vinculada al cambio de gobierno y refleja un modo muy específico de entender la política exterior, alejada del cariz cosmopolita que tuvo la política exterior socialista.

Pero, ¿qué sucede cuando analizamos la priorización y el abordaje en ambas políticas de las cuestiones de género? ¿Qué transformaciones ligadas a la crisis y al cambio de gobierno se han producido en relación al alineamiento de estas políticas con los compromisos internacionales de género asumidos por el Estado español?

A grandes líneas, la diferente priorización de la igualdad de género en la cooperación española y en la iniciativa Marca España refleja tanto cambios ligados a la crisis como otro tipo de transformaciones que bajo la etiqueta de "medidas a impulsar por la crisis" esconden apuestas políticas que no se alinean con los compromisos internacionales de género y pueden tener efectos perversos sobre los pasos ya avanzados en materia de desarrollo pro-equidad de género.

Así, en el período analizado, la igualdad de género conforma un objetivo clave de la cooperación española y, en el discurso, se mantiene el compromiso con el mainstreaming de género y el empoderamiento de las mujeres, en línea con la Conferencia de Beijing. No obstante, en el último Plan Director, se ha puesto un foco específico en las mujeres como agentes clave en el crecimiento económico que 
corre el riesgo de generar sobrecarga laboral en las mismas y de excluir a hombres y niños de estos procesos de cambio perdiendo potencialidad sus posibles impactos.

Si dirigimos la mirada a la iniciativa Marca España, sin embargo, la igualdad de género ni siquiera se considera como un tema a abordar. Por el contrario, se entiende como un tema superado y, a este respecto, se aleja de los compromisos internacionales asumidos por el Estado español en esta materia que de la mano de la estrategia de mainstraming de género- reconocen la necesidad de incorporar la igualdad de género como un contenido central en toda la agenda política. De forma paralela, la iniciativa Marca España se orienta a impulsar la imagen-país para favorecer la salida a la crisis a través de las exportaciones y la internacionalización de la empresa como dos de sus elementos clave. Y, en este sentido, se encuentra en línea con la agenda neoliberal y del laissez faire que asume que el crecimiento económico tendrá efectos beneficiosos sobre las todas las personas. Empero, obvia los impactos diferenciados de género de la actividad empresarial y el peligro de fomentar el crecimiento de las empresas españolas apoyándose en las ventajas comparativas que genera la división sexual del trabajo. Asimismo, desde una estrategia de promoción de imagen-país, desaprovecha el camino avanzado por los anteriores gobiernos socialistas en la proyección internacional del Estado español como defensor de los derechos de las mujeres y de la igualdad de género.

En cuanto al abordaje de la igualdad de género en ambas políticas, el mismo revela situaciones diferentes marcadas, en todo caso, por una escasa centralidad de la igualdad que también aleja al Estado español de los compromisos internacionales firmados en esta materia. En efecto, en la iniciativa Marca España no existe ningún tipo de estructura, recurso o mecanismo para promover la igualdad, en línea con la ceguera al género de esta iniciativa. En la cooperación española, aunque en el discurso se mantiene el compromiso con la igualdad, se han experimentado reducciones en los fondos destinados a sectores críticos de género, cambios en la ubicación de los contenidos de género en su organigrama y cierre de programas que ponen en peligro los avances efectivos en materia de igualdad.

En cuanto a la AOD destinada a sectores críticos de género, la caída de la ayuda dirigida a organizaciones e instituciones de igualdad y a salud sexual y reproductiva ya comienza en la última legislatura del gobierno de Rodríguez Zapatero. En todo caso, es con el Gobierno popular cuando la Unidad de Género de la AECID pierde centralidad en el organigrama, se decide el cierre de la oficina de ONU Mujeres en el Estado español y se deja de financiar la Red de Mujeres Africanas y Españolas, un proyecto definidor de la apuesta de la cooperación española por la igualdad en anteriores años. Asimismo, las medidas políticas impulsadas en otras áreas -como igualdad, educación, violencia de género, entre 
otras- por parte del gobierno del Partido Popular revelan cambios ligados no sólo a la crisis sino al partido en el poder y hace que tengamos que estar vigilantes de sus acciones en esta dirección para evitar que los compromisos con la igualdad se evaporen en la práctica de la cooperación y sean usados de forma cosmética.

Todo esto revela unas serias inconsistencias por parte del Estado español a la hora de promover un desarrollo pro-equidad de género. Unas inconsistencias que, como hemos señalado, puede tener el efecto de generar efectos contradictorios: mientras que desde la cooperación española se fomenta -con reducidos recursos y mecanismos- la igualdad de género, desde la Marca España se apoya una internacionalización de la empresa que saca provecho de la división sexual del trabajo. Éste es, sin duda, un campo en el que seguir indagando de cara a promover, en efecto, el desarrollo de mujeres y hombres a lo largo de todo el planeta.

\section{BIBLIOGRAFÍA}

ALONSO, Alba y POLEO, Natalia (2013): “¿Es únicamente una cuestión de austeridad? Crisis económica y políticas de género en España". Paper presentado en XI Conferencia AECPA. Sevilla.

ATIENZA, Jaime y HERNANDEZ, Verónica (2012): "Desmantelando el futuro. La cooperación internacional, al borde del abismo", en La realidad de la ayuda 2012. Barcelona. Intermón Oxfam.

BUSTELO, María y LOMBARDO, Emanuela (2007): “¿Qué hay debajo de la alfombra de las políticas de igualdad? Un análisis de "marcos interpretativos" en España y en Europa" en María Bustelo y Emanuela Lombardo (eds.) Políticas de igualdad en España y en Europa. Madrid. Cátedra, Universitat de Valencia e Instituto de la Mujer, Ministerio de Trabajo y Asuntos Sociales. Pp. 11-35.

CIRUJANO CAMPANO, Paula (2005): La estrategia demorada. Género y cooperación internacional en España. Madrid. Editorial La Catarata.

ESPINOSA FAJARDO, Julia y GALLARDO GARCÍA, Begoña (2013): Coherencia de políticas y género. Un análisis feminista de la promoción del desarrollo internacional. Madrid: Plataforma 2015 y más. Disponible en: http://2015ymas.org/centro-dedocumentacion/publicaciones/2013/1557/coherencia-de-politicas-ygenero/\#.Ut4uT uCEdU

ESPINOSA FAJARDO, Julia (2011): La igualdad de género en la evaluación de la ayuda: los casos de la cooperación oficial británica, sueca y española. Tesis doctoral. Madrid. Universidad Complutense de Madrid. Disponible en: http://eprints.ucm.es/13206/

ESPINOSA FAJARDO, Julia (2005): Guía para programas y proyectos de salud sexual y reproductiva en África. Madrid. Programa Vita, Agencia Española de Cooperación Internacional para el Desarrollo. Disponible en: 
http://fcsai.isciii.es/formacion/pdf/Guia para Programas y Proyectos Salud Sexual y Reproductiva en Africa.pdf

FERNÁNDEZ-LAYOS FERNÁNDEZ, Ana Lydia (2013): "España también recorta en igualdad", en Diario Atlántida. Disponible en: http://www.diarioatlantida.com/igualdad/espana-tambien-recorta-en-igualdad

FLORO, Maria y HOPPE, Hella (2005): Engendering Policy Coherence for Development: Gender Issues for the Global Policy agenda in the year 2005, Berlín. Fundación Friedrich Ebert. Disponible en: http://library.fes.de/pdffiles/iez/global/50085.pdf

GRUPO MAYOR DE MUJERES (2013): Fortalecimiento de la justicia de género. Recomendaciones para los Objetivos de Desarrollo Sostenible y la Agenda para el Desarrollo Post 2015. Friedrich-Ebert-Stiftung.

GRUPO DE TRABAJO DE NACIONES UNIDAS PARA LA AGENDA DE DESARROLLO POST 2015 (2012): El futuro que queremos para todos. ONU. http://www.un.org/en/development/desa/policy/untaskteam undf/unttreport sp. pdf

INTERMÓN OXFAM (2013): La empresa española y los Derechos Humanos. Claves para una internacionalización responsable. Disponible en: http://imagenes.publico.es/resources/archivos/2013/6/11/1370971806384Infor meIOE.pdf

JUBETO, Yolanda (2011): "Debates sobre desarrollo y bienestar desde la economía feminista". Revista Pueblos, $\mathrm{n}^{\circ}$ 49, especial diciembre.

KABEER, Naila (2005): "Gender Equality and Women's Empowerment: A Critical Analysis of the Third Millennium Development Goal", Gender and Development, 13(1), pp. 13-24.

LEÓN, Magdalena (2008): "Cambiar la economía para cambiar la vida", en Alberto Acosta y Esperanza Martínez (comp.), El buen vivir: una vía para el desarrollo. Quito. Ed. Abya-Yala. Disponible en http://www.fedaeps.org/IMG/pdf/CAMBIAR LA ECONOMIA PARA CAM BIAR_LA_VIDA.pdf

LOMBARDO, Emanuela (2013): "Gender mainstreaming and policy responses to the economic crisis: the 'unintended consequences' of EU and national policymaking on Spanish gender equality policies". Paper presentado en XI Conferencia AECPA. Sevilla. En proceso de evaluación por una revista peerreviewed.

MARTÍNEZ, Pablo y MARTÍNEZ, Ignacio (2014): "Ruptura del consenso y desmantelamiento: un análisis de la cooperación española" en XI Anuario de la Plataforma 2015 y más. Madrid. Editorial 2015 y más. Disponible en http://www.2015ymas.org/centro-dedocumentacion/publicaciones/2014/1576/ruptura-del-consenso-ydesmantelamiento-un-analisis-de-la-cooperacion-espanola/\#.U70VjkCs-6U

MILLÁN, Natalia, SANTANDER, Guillermo, AGUIRRE, Pablo y GARRIDO, Anabel (2012): La coherencia de políticas para el desarrollo en España. Madrid. Editorial 2015 y más. 
MILLÁN ACEVEDO, Natalia (2013): “Marca España: ¿qué implicaciones tiene para el desarrollo humano?, en Cuadernos 2015 y más, nº 1 . Madrid. Editorial 2015 y más.

MORO-COCO, Mayra y RAABER, Natalie (2013): Llegando hasta la raíz: reincorporación de los Derechos Humanos y la igualdad de género en la Agenda de Desarrollo Post 2015. AWID. Disponible en: http://www.awid.org/esl/Media/Getting-at-the-roots

MOSER, Caroline O.N. (1995): Planificación de género y desarrollo. Teoría, práctica y capacitación. Lima. Red Entre Mujeres y Flora Tristán Ediciones.

MUKHOPADHYAY, Maitrayee (2007): "Mainstreaming gender or "streaming" gender away: feminists marooned in the development business", en Andrea Cornwall, Elizabeth Harrison and Ann Whitehead (eds.) Feminisms in Development: Contradictions, Contestations \& Challenges. Londres. Zed Books.

NOYA MIRANDA, Javier (2009): La nueva imagen de España en América Latina. Madrid. Tecnos y Real Instituto El Cano.

OBSERVATORIO DE RESPONSABILIDAD SOCIAL CORPORATIVA (2012): Informe anual del Observatorio de Responsabilidad Social Corporativa. Disponible http://www.observatoriorsc.org/Informe memoriasRSC ibex 2012 final com pleto.pdf

PEARSON, Ruth (1999): "El género cuenta en el desarrollo" en Cristina Carrasco (Ed.) Mujeres y economía. Nuevas perspectivas para viejos y nuevos problemas. Barcelona. Icaria. Pp. 365-398.

PÉREZ GONZÁLEZ, Javier y FANJUL, Gonzalo (2012): Hacia un libro blanco de la política española de desarrollo. Madrid. CIECODE, Fundación Salvador Soler - Mundo Justo.

SALVADOR, Soledad (2012): “Género y Comercio en América Latina” en Valeria Esquivel (Ed.) La economía feminista desde América Latina. Una hoja de ruta sobre los debates actuales en la región. ONU MUJERES.

WILlIAMS, Mariama (2003): Gender Mainstreaming in the Multilateral Trading System. Londres. Commonwealth.

WOMEN'S COALITION (2014): “The Post 2015 Development Agenda: What's at Stake for the World's Women?".Women's Coalition. Disponible en: http://www.eldis.org/go/home\&id=67908\&type=Document\#.U8PID0Cs-6U

WOMEN WATCH (2011): Gender Equality \& Trade Policy. Resource Paper. Disponible

en: http://www.un.org/womenwatch/feature/trade/gender equality and trade polic y.pdf 


\section{Documentación consultada}

\section{Compromisos internacionales}

ONU (2010): Resolución de la Asamblea General Sexagésimo $5^{\circ}$ Período de sesiones de la Cumbre de los objetivos de desarrollo del Milenio "Cumplir la promesa: unidos para lograr los Objetivos de Desarrollo del Milenio”.

ONU (2000): Resolución de la Asamblea General en el Quincuagésimo quinto período de sesiones por la que se aprueba la Declaración de la Cumbre del Milenio.

ONU (1999): Resolución A/54/4 de la Asamblea General para la adopción del Protocolo a la Convención para la Eliminación de Todas las Formas de Discriminación Contra la Mujer.

ONU (1995): Resolución de la $4^{a}$ Conferencia Mundial de la Mujer por la que se aprueba La Declaración y Plataforma para la Acción de Beijing.

ONU (1993): Declaración y Programa de Acción de Viena. Conferencia Mundial de Derechos Humanos 157/23.

ONU (1979): Resolución 34/180 de la Asamblea General para la adopción de la Convención sobre la Eliminación de todas las Formas de Discriminación contra la Mujer. CEDAW.

ONU (1976): Resolución 2200 de la Asamblea General para la adopción del Pacto Internacional de Derechos Económicos, Sociales y Culturales.

\section{Normativa nacional}

BOE (2012a): Real Decreto 998/2012, de 28 de junio, por el que se crea el Alto Comisionado del Gobierno para la Marca España y se modifica el Real Decreto 1412/2000, de 21 de julio, de creación del Consejo de Política Exterior.

BOE (2012b): Real Decreto 1424/2012, de 11 de octubre, por el que se modifica el Estatuto de la Agencia Española de Cooperación Internacional para el Desarrollo, aprobado por Real Decreto 1403/2007, de 26 de octubre, se adecuan otros órganos colegiados en materia de cooperación y se modifica el Reglamento del Instituto Cervantes, aprobado por Real Decreto 1526/1999, de 1 de octubre.

BOE (1998): Ley 23/1998 del 7 de julio de Cooperación Internacional para el Desarrollo.

ESCUELA DIPLOMÁTICA (2012): Retos de nuestra acción exterior: Diplomacia Pública y Marca España. Colección Escuela Diplomática, no 18. Madrid. Ministerio de Asuntos Exteriores y Cooperación.

MAE (2005): Plan Director de la Cooperación Española 2005-2008. Madrid, MAESECI.

MAEC (2012): Plan Director de la Cooperación Española 2013-2016. Madrid, MAEC-SECI.

MAEC (2009): Plan Director de la Cooperación Española 2009-2012. Madrid, MAEC-SECI.

MAEC (2007): Estrategia de Género en Desarrollo de la Cooperación Española. Madrid, MAEC y SECI. 


\section{Anexo. Preguntas guía}

Código:

Título completo del texto

Fecha de publicación

Partido político en el Gobierno

Voz:

Voces presentes y ausentes en el texto

Diagnóstico:

¿Qué se identifica como problema cuando se habla de desarrollo?

¿En qué medida el género se vincula con este problema?

¿Qué dimensiones o temas críticos de género son incluidos en este diagnóstico?

¿Qué dimensiones o temas críticos de género son excluidos en este diagnóstico?

Pronóstico:

¿Qué acciones se consideran necesarias para promover el desarrollo?

¿Quiénes se benefician de la política de cooperación al desarrollo?

¿Qué papel tiene la igualdad de género en el seno de la política analizada?

¿Qué estrategias se definen para promover un desarrollo pro-igualdad de género?

¿Y qué acciones se impulsan en esta dirección?

¿Qué tipo de instrumentos y recursos para promover la igualdad se definen en las políticas señaladas? 